\title{
Optimal Grip Span of A-type Pliers in a Maximum Gripping Task
}

\author{
Yong-Ku Kong ${ }^{1}$, Jin Woo Jung ${ }^{1}$, Sangmin Kim ${ }^{1}$, Heewoong Jung ${ }^{1}$, \\ Hakje Yoo ${ }^{2}$, Dae-Min Kim ${ }^{1}$, Hyun-Sung Kang ${ }^{1}$ \\ ${ }^{1}$ Department of Industrial Engineering, Sungkyunkwan University, Suwon, 440-746 \\ ${ }^{2}$ Department of Biomechatronics, Sungkyunkwan University, Suwon, 440-746
}

\begin{abstract}
Objective: The objective of this study is designing an optimal hand tool through maximum grip force study accordance to the hand grip span. Background: In order to prevent musculoskeletal diseases, studies on hand tool design are proceeding based on grip strength, finger force, and contribution of individual finger force on total grip strength. However, experimental apparatus using a tool that is actually used in work place was almost non-existent. Method: 19 males were participated in an experiment. Using the load cell inserted real plier, finger force, grip strength, and subjective discomfort rate of both hands (dominant and non-dominant) were measured in 5 different hand grip span $(45 \mathrm{~mm}, 50 \mathrm{~mm}, 60 \mathrm{~mm}, 70 \mathrm{~mm}$, and $80 \mathrm{~mm}$ ). Results: There was significant difference $(p<0.001)$ of total grip strength, individual finger force and subjective discomfort rating according to various hand grip $\operatorname{span}(45,50,60,70$, and $80 \mathrm{~mm})$. Also, statistically significant $\operatorname{different}(p<0.001)$ was shown between the dominant hand and non-dominant hand. In addition, individual finger force in maximum grip was in order of middle finger, ring finger, index finger, and little finger. Conclusion: Optimal grip span of pliers that exerting maximum grip strength is 50 60mm. Application: This finding is expected to be used for designing proper pliers.
\end{abstract}

Keywords: A-type hand tools, Pliers, grip span, Maximum grip, Subjective discomfort rate

\section{Introduction}

대부분의 산업 현장에서 반복적인 작업으로 인해 작업자 의 신체 특정 부위에 부하가 발생되며 이는 근골격계 질환 으로 이어질 가능성이 높다. 그에 따라 신체 특정 부위, 그 중에서도 손에 대한 부하를 줄이기 위한 수공구의 최적 설계 에 대한 연구가 지속되고 있다.

물체를 쥐는 힘인 악력은 수공구 설계 관점에서 손 관련 근골격계 질환 예방을 위한 중요한 고려 요소이다. 악력에 대한 연구는 파지 폭, 파지 형태, 각 손가락 별 힘, 주력 손과 비주력 손 등 여러 가지 방향으로 접근하여 수행되어 왔다.
관련된 선행 연구로 최대 악력을 발휘하는 최적의 파지 폭에 대한 연구가 수행되었으며(Petrofsky et al., 1980; Fransson and Winkel, 1991; Talsania and Kozin, 1998; Eksioglu, 2004), 특히 Kong et al.(2008)의 연구에서는 전체 힘 뿐만 아닌 각 손가락의 힘을 측정하여 5 가지 파지 폭에 대한 최대 악력 수행 시 파지 폭에 따라 총 악력 뿐만 아니라 손가락 별 악력의 유의한 차이를 확인하였다.

파지 폭에 따라 손가락의 힘이 달라지는 점에 미루어 보았 을 때, 악력은 파지 형태에 따라서 달라질 것이라 생각할 수 있는데 Kong et al.(2009)의 연구에서는 파지 형태를 검지 와 소지의 파지 폭에 따라 A, D, I, V 네 가지 형태로 분류하 였고, Fransson and Winkel(1991)의 연구에서는 플라이어

Corresponding Author: Hyun-Sung Kang. Department of Industrial Engineering, Sungkyunkwan University, Suwon, 440-746. Phone: +82-31-290-7629, E-mail: kanghs328@naver.com Copyright@2013 by Ergonomics Society of Korea(pISSN:1229-1684 eISSN:2093-8462). All right reserved.

(c) This is an open-access article distributed under the terms of the Creative Commons Attribution Non-Commercial License(http://creativecommons.org/licenses/by-nc/3.0/), which permits unrestricted non-commercial use, distribution, and reproduction in any medium, provided the original work is properly cited. http://www.esk.or.kr 
를 잡는 형태에 따라 검지가 파지되는 부분보다 소지가 파지 되는 부분의 길이가 긴 traditional grip과 검지 파지 폭보다 소지 파지 폭이 작은 reversed grip으로 분류하였다. 이중 A형 손잡이 (traditional grip)에 대한 연구로 Greenberg and Chaffin(1977) 과 Fransson and Winkel(1991)의 연 구가 존재한다. 각 연구에서 최적의 파지 폭은 $75 ~ 80 \mathrm{~mm}$ (Greenberg and Chaffin, 1977), 남성 55 65mm, 여성 50 60mm (Fransson and Winkel, 1991)이라 하였다. 각 손가락 별 힘을 측정할 수 있는 MFFM system (Kong et al., 2008)의 확장 연구로 제작한 A형 손잡이에 대한 Kong et al. (2011)의 연구에서는 동일한 장비의 I 타입과 다소 다른 최적 파지 폭을 도출하였다.

최대 악력을 발휘할 수 있는 최적의 파지 폭과 관련해, 최 대 악력 과업 수행 시 각 손가락에서 발생하는 힘에 대한 기 존 연구 결과를 보면 대부분의 연구에서 중지가 가장 큰 힘 을 나타내었고 다음으로 검지와 약지의 힘이 비슷하거나 작 은 차이가 존재하였으며 소지는 가장 약한 힘을 발휘하였다 (Hazelton et al., 1975; Fransson and Winkel, 1991; Farris et al., 1997). 하지만 본 파지 형태가 달라짐에 따라 기존 I형 손잡이에서 나타나는 손가락 별 힘의 비율과 유사한 결 과를 나타낼 것인가를 고려해 보아야 한다. 각 손가락에서 발휘되는 힘의 크기가 파지 폭에 따라 다른 만큼 파지 형태 의 변화에 따라서 각 손가락에서 발생하는 힘의 비율이 변할 수 있기 때문이다.

본 연구에서는 실제 공구 활용 시 발생하는 손가락 힘과 악력, 그리고 주관적 불편도를 이용해 최적의 플라이어 파지 폭을 도출하려고 한다. 실험 장비는 일반적으로 작업 현장에 서 많이 사용되는 A 타입의 플라이어를 사용하여 각 손가락 힘을 측정할 수 있도록 개조하여 사용하였다.

\section{Method}

\subsection{Subjects}

본 연구를 위해 과거 및 현재 상지에 근골격계관련 질환 병력이나 기타 질환이 없는 성인 남성 19 명이 참가하였다. 실험 참가자의 평균 나이는 $25.7 \pm 2.5$ 세, 키 $176.0 \pm 3.6 \mathrm{~cm}$, 몸무게 $69.7 \pm 8.2 \mathrm{~kg}$, 손 길이 $18.8 \pm 0.9 \mathrm{~cm}$, 손 너비 $8.2 \pm$ $0.5 \mathrm{~cm}$ 이다.

\subsection{Equipment}

일반적인 작업 현장에서 사용되는 플라이어의 헤드 부분 과 손잡이 경계 부분의 구조를 개조하여 파지 폭을 자유로
이 조절할 수 있도록 하였고, 각 손가락의 힘을 측정할 수 있도록 파지하는 부분에 로드셀을 삽입한 플라이어를 제작 하였다(Figure 1). 플라이어의 파지 폭은 로드셀이 장착된 손잡이의 중지의 중점 부분을 기준으로 총 5 가지 $(45 \mathrm{~mm}$, $50 \mathrm{~mm}, 60 \mathrm{~mm}, 70 \mathrm{~mm}, 80 \mathrm{~mm}$ )를 선정하였다(Figure 2). 모든 데이터는 LabVIEW 프로그램을 사용하여 측정하고 저 장하였다.

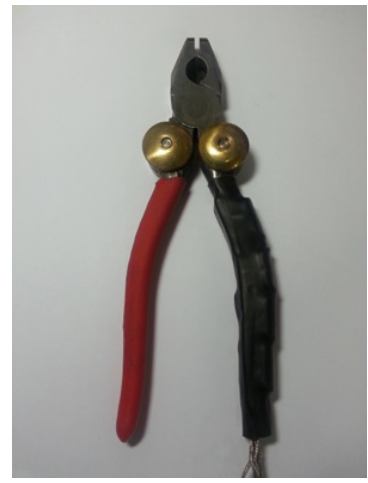

Figure 1. Custom-made pliers

\begin{tabular}{c|c|c|c|c|c}
\hline \multicolumn{1}{|c|}{} & \\
\hline & $45 \mathrm{~mm}$ & $50 \mathrm{~mm}$ & $60 \mathrm{~mm}$ & $70 \mathrm{~mm}$ & $80 \mathrm{~mm}$ \\
\hline Index & 40.74 & 45.79 & 51.52 & 57.66 & 62.00 \\
\hline Middle & 44.54 & 52.20 & 60.80 & 69.74 & 80.05 \\
\hline Ring & 39.51 & 49.79 & 62.60 & 72.29 & 86.51 \\
\hline Little & 30.81 & 44.00 & 60.20 & 72.05 & 90.36 \\
\hline
\end{tabular}

Figure 2. Specification of custom-made pliers

\subsection{Experimental design}

독립변수로 파지 폭 $(45 \mathrm{~mm}, 50 \mathrm{~mm}, 60 \mathrm{~mm}, 70 \mathrm{~mm}$, $80 \mathrm{~mm}$ ) 과 주력 손, 그리고 손가락(검지, 중지, 약지, 소지) 을 선정하였으며, 종속변수로 총 악력과 각 손가락 힘, 그리 고 10점 척도의 주관적 불편도(VAS: Visual Analog Scale) 를 선정하였다.

통계적 분석은 SPSS (ver. 18.0)을 활용하여, ANOVA를 수행하였으며, 다중비교 방법은 Tukey HSD를 선택하여 유 의수준 $\alpha=0.05$ 로 검증하였다. 


\subsection{Experimental procedures}

실험 전, 상지 근골격계 질환 및 실험에 영향을 미칠 신체 질환에 대한 응답지를 작성한 후, 실험 참가자의 정보 및 신 체 치수를 측정하였다. 다음으로 실험 자세와 실험 방법에 대해 설명한 후, 플라이어에 대한 파지 방법과 사전 연습을 수행하였다. 악력 측정은 주력 손과 비주력 손을 모두 사용 하여 수행하였다. 본 실험에서는 1 회당 약 5 초간 악력을 측 정하였으며, 각 측정 사이에 약 3 분 간의 휴식 시간을 제공 하여 손과 측정 근육의 피로도를 최소화하였다. 측정 후 손 의 각 부위에 대한 주관적 불편도를 VAS scale을 이용하여 평가하였으며, 양 손에 대해 무작위로 5 가지 파지 폭에서 각 2 회 반복하여 총 20 회의 최대 악력을 측정하였다.

\section{Results}

\subsection{Grip strength}

파지 폭에 따라 총 악력은 통계적으로 유의한 차이를 보였 고 $(p<0.001)$, 각 손가락의 악력도 통계적으로 유의한 차이 를 나타냈으며 $(p<0.001)$, 주력 손과 비주력 손의 차이 역 시 통계적으로 유의한 차이를 보였다 $(p<0.001)$.

총 악력은 $60 \mathrm{~mm}$ 의 파지 폭에서 $345 \mathrm{~N}$ 으로 가장 높은 악 력을 보였고, $80 \mathrm{~mm}$ 의 파지 폭에서 $261 \mathrm{~N}$ 으로 가장 낮은 악 력을 보였다(Figure 3).

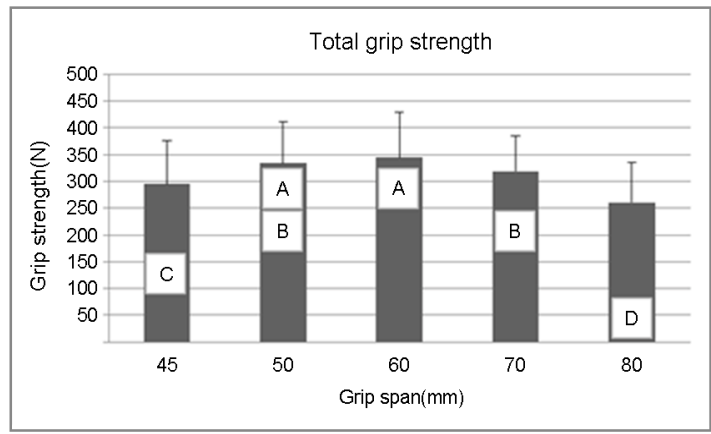

Figure 3. Total grip strength over grip spans

최대 악력의 손가락 별 힘에서 가장 높은 힘을 보인 손가 락은 중지 $(109 \mathrm{~N})$ 이고, 다음으로 약지 $(84 \mathrm{~N})$, 검지 $(73 \mathrm{~N})$, 소지 $(45 \mathrm{~N})$ 순으로 각각 통계적으로 유의한 차이를 나타냈 다 $(p<0.001)$ (Figure 4$)$

주력 손과 비주력 손에 대한 최대 악력은 주력 손 $323 \mathrm{~N}$,

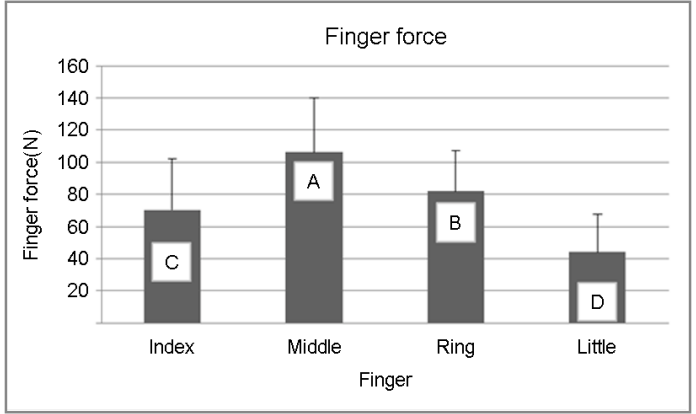

Figure 4. Individual finger force in maximum grip strength

비주력 손 $299 \mathrm{~N}$ 으로 통계적으로 유의한 차이를 나타냈으며 ( $p<0.001)$, 주력 손에 대해 비주력 손의 악력이 $92.6 \%$ 의 악력을 나타냈다.

파지 폭과 손가락 악력 간의 교호작용 역시 Figure 5 와 같이 통계적으로 유의하였다 $(p<0.001)$. 파지 폭과 손가락 간의 교호작용에서, 검지와 중지의 파지 폭이 증가할수록 각 손가락에서 발생하는 힘이 통계적으로 유의하게 감소하 였고, 약지와 소지의 힘은 통계적으로 유의하게 증가하였다 (Table 1).

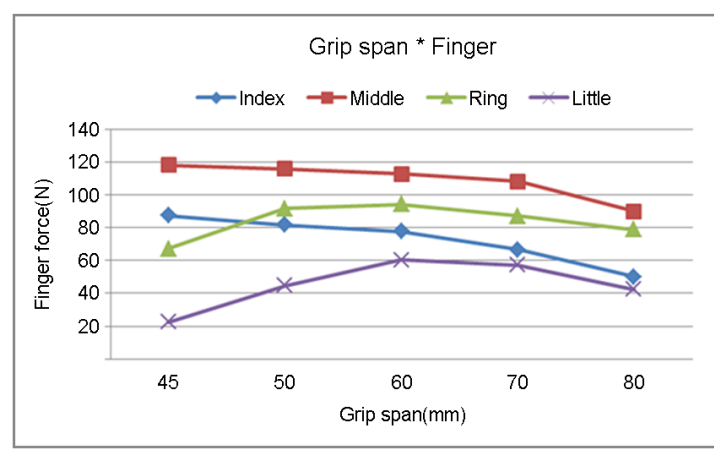

Figure 5. Interaction of finger and grip span

Table 1. Individual finger force over grip span

\begin{tabular}{c|c|c|c|c|c}
\hline & $45 \mathrm{~mm}$ & $50 \mathrm{~mm}$ & $60 \mathrm{~mm}$ & $70 \mathrm{~mm}$ & $80 \mathrm{~mm}$ \\
\hline Index & $87 \pm 34^{\mathrm{A}}$ & $82 \pm 28^{\mathrm{A}}$ & $78 \pm 28^{\mathrm{AB}}$ & $67 \pm 26^{\mathrm{B}}$ & $50 \pm 22^{\mathrm{C}}$ \\
\hline Middle & $118 \pm 35^{\mathrm{a}}$ & $116 \pm 30^{\mathrm{a}}$ & $113 \pm 31^{\mathrm{a}}$ & $108 \pm 28^{\mathrm{a}}$ & $90 \pm 30^{\mathrm{b}}$ \\
\hline Ring & $67 \pm 21^{\gamma}$ & $92 \pm 24^{\alpha}$ & $94 \pm 25^{\alpha}$ & $87 \pm 20^{\alpha \beta}$ & $79 \pm 22^{\beta}$ \\
\hline Little & $22 \pm 13^{\mathbf{C}}$ & $44 \pm 19^{\underline{\mathbf{B}}}$ & $60 \pm 24^{\underline{\mathbf{A}}}$ & $57 \pm 22^{\mathbf{A}}$ & $42 \pm 15^{\underline{\mathbf{B}}}$ \\
\hline
\end{tabular}

손가락과 주력 손의 교호작용은 통계적으로 유의하였지만 모든 손가락에서 주력 손이 비주력 손보다 높은 악력을 나타 
냈다(Figure 6).

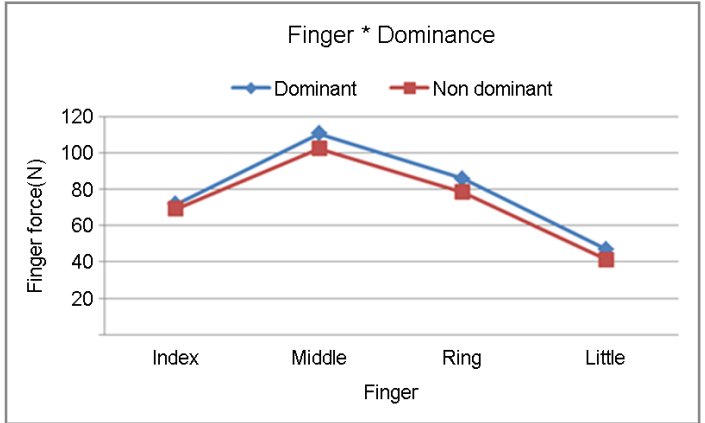

Figure 6. Interaction of finger and dominant hand

\subsection{Subjective discomfort rating}

최대 악력 측정 시, $\mathrm{VAS}$ 을 활용하여 측정한 손의 주관적 불편도는 파지 폭에 따라 통계적으로 유의한 차이를 보였다 $(p<0.001)$.

Figure 6을 보면, $60 \mathrm{~mm}$ 파지 폭에서 가장 낮은 주관적 불편도를 나타냈으며 $80 \mathrm{~mm}$ 의 파지 폭에서 가장 높은 주관 적 불편도를 나타냈다(Figure 7).

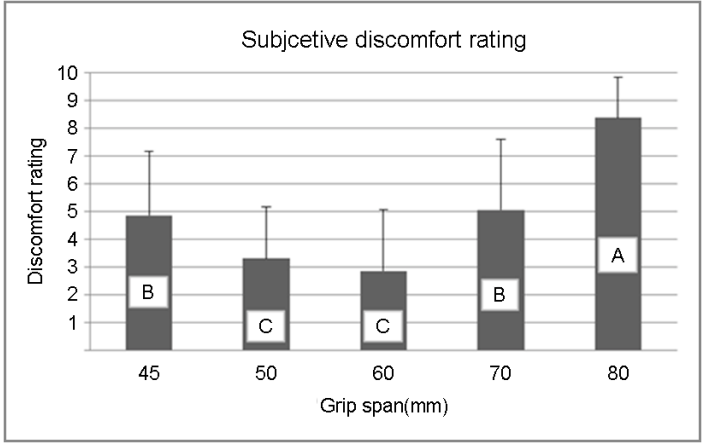

Figure 7. Subjective discomfort rating over grip spans

\section{Discussion}

본 연구에서는 플라이어의 파지 폭에 대한 최대 악력 및 각 손가락(검지, 중지, 약지, 소지)의 힘, 그리고 주관적 불 편도를 측정하여 분석하였다.
실험에서 사용된 장비는 실제 작업에서 활용되는 플라이 어를 개조한 것으로 헤드 부분과 손잡이 경계 부분을 변형 하여 파지 폭이 조절 가능하며 동시에 손가락 별 악력을 측 정할 수 있도록 만들어졌다. 악력 및 손가락 힘 측정을 위한 기존 연구에서의 개발 장비를 보자면, 먼저 각 손가락의 힘 을 측정한 장비가 있다.

본 연구에서 사용된 실험 장비는 실제 플라이어의 형태를 가지며 동일한 기능을 수행할 수 있기 때문에 실제 작업에서 각 손가락의 힘을 측정할 수 있다. 또한 파지 폭을 조절을 통해 실제적 연구와 실험적 연구를 동시에 수행할 수 있다.

본 연구에서 분석된 총 악력에 대한 파지 폭에 따른 최대 악력에 대한 기존 연구의 결과와 유사한 결과를 보인다. 파 지 폭에 따른 각각의 손가락 힘 사이의 관계를 보자면, 총 악력은 $60 \mathrm{~mm}$ 의 파지 폭에서 $345 \mathrm{~N}$ 으로 가장 높은 악력을 보이며, $80 \mathrm{~mm}$ 의 파지 폭에서 $261 \mathrm{~N}$ 으로 가장 낮은 악력을 보였다. $45 \mathrm{~mm}$ 에서 $60 \mathrm{~mm}$ 까지 파지 폭 증가에 따라 총 악 력은 증가하였고 이후 $80 \mathrm{~mm}$ 까지는 감소하였다. 이는 최대 악력을 발휘하는 데 적정 수준의 파지 폭이 존재한다고 할 수 있으며 일정 수준의 파지 폭 범위에서 벗어나면 최대 악 력은 감소한다고 할 수 있다.

이 결과는 기존 $\mathrm{A}$ 형 손잡이에서 남성의 최적 파지 폭 은 55 65mm라는 Fransson and Winkel(1991)의 연구 결과와 유사하며, $75 \sim 80 \mathrm{~mm}$ 가 최적의 파지 폭이라는 Greenberg and Chaffin(1977)의 결과와는 다소 차이가 있 다. 하지만 실험을 위한 장비가 아닌 실제 사용하는 수공구 를 이용한 결과인 Fransson and Winkel(1991)의 연구 결 과와의 유사성을 볼 때, 실제 수공구에 적용 가능할 수 있을 것으로 생각된다.

다음으로 손가락 별 힘을 보면 가장 높은 힘을 보인 손가 락은 중지 $(109 \mathrm{~N})$ 이고, 다음으로 약지 $(84 \mathrm{~N})$, 검지 $(73 \mathrm{~N})$, 소지 $(45 \mathrm{~N})$ 순으로 각각 통계적으로 유의한 차이를 나타냈 다. 총 악력에 대해 각 손가락에서 발생하는 악력의 기여도는 모든 파지 폭에서 평균적으로 중지 (35.0\%), 약지(27.0\%), 검지 $(23.5 \%)$, 소지 $(14.5 \%)$ 순으로 나타났다. 기존 연구에 따르면 총 악력에 대한 손가락 별 악력의 기여도는 중지가 가장 크고 소지가 가장 작으며 검지와 약지가 비슷하거나 차 이를 보이는 경우가 존재한다. 본 연구에서는 약지의 기여도 가 검지의 기여도보다 높게 나왔는데, 손가락 별 힘을 측정 한 A-type 수공구의 Fransson and Winkel(1991)의 연구 결과에서 약지가 총 악력에 기여하는 기여도는 $26.5 \%$ 로 이 역시 기존 연구 결과와 유사하다.

주력 손과 비주력 손의 총 악력은 통계적으로 유의한 차 이를 보였으며 $(p<0.001)$, 최대 악력은 주력 손 $323 \mathrm{~N}$, 비 주력 손 $299 \mathrm{~N}$ 으로, 주력 손에 대해 비주력 손의 악력이 $92.6 \%$ 의 악력을 나타냈다. 또한 손가락과 주력 손의 교호작 
용은 통계적으로 유의하였다.

주관적 불편도 역시 파지 폭에 따른 통계적으로 유의한 차 이를 보였다 $(p<0.001)$. 가장 큰 불편도를 나타낸 파지 폭 은 $80 \mathrm{~mm}$ 였으며, $45 \mathrm{~mm}, 70 \mathrm{~mm}$ 의 파지 폭은 그 보다 낮은 불편함을 느꼈다. 가장 불편함 없는 파지 폭 그룹은 $50 \mathrm{~mm}$ 와 $60 \mathrm{~mm}$ 이다. Figure 7 과 같이 주관적 불편도와 최대 악력 간의 관계를 보았을 때, 가장 불편도가 낮은 $50 \mathrm{~mm}$ 파지 폭 에서 가장 큰 총 악력 값이 나타났으며, 주관적 불편도 높은 $80 \mathrm{~mm}$ 에서는 가장 작은 총 악력이 측정되었다. 이를 통해, 피실험자가 최대 악력 과업 수행 시 느끼는 불편도는 충분히 큰 힘을 발휘할 경우 조금 불편하다고 느끼고 본인이 낼 수 있는 힘을 제대로 발휘할 수 없을 경우 불편하다고 느끼는 것이라 할 수 있다.

\section{Conclusions}

본 연구는 상지 관련 근골격계 병력이 없는 성인 남성 19 명을 대상으로 기존에 작업 현장에서 사용되는 플라이어 를 각 손가락 별 악력을 측정할 수 있도록 개조하여 최대 악 력 실험을 수행하였다. 실험을 통하여 총 악력 및 손가락 힘, 주력 손의 차이, 그리고 주관적 불편도를 파악하여 다음과 같은 결론을 도출하게 되었다.

총 악력은 50 60mm의 파지 폭에서 통계적으로 가장 높 게 나타났고, 손가락 별로 발휘하는 힘에 대해 통계적으로 유의한 차이가 존재하였다. 주력 손이 비주력 손보다 큰 악 력을 나타냈으며, 주관적 불편도는 파지 폭에 따라 차이가 존재하여, 가장 큰 악력을 내는 $60 \mathrm{~mm}$ 파지 폭에서 가장 낮 은 주관적 불편도를 나타내, 가장 큰 악력을 발휘하는 파지 폭에서 손이 편안함을 확인할 수 있었다.

본 연구에서 개발된 플라이어는 실제 상용 제품을 개조하 여 현장에서 수행하는 다양한 작업 동작에 대해 분석할 수 있는 장점이 있으며, 본 연구 결과는 그 기초 데이터로 최대 악력 수행시의 파지 폭을 확인하여 기존 연구와 비교할 수 있었다.

연구의 한계점으로는 사회적 제약으로 인해 비교적 양 손 의 악력 차이가 작은 왼손잡이의 특성을 고려하지 않았다는 점이다. 피실험자 수를 늘려, 주력 손이 오른손인 내국인을 대상으로 실험을 한 결과가 추가적으로 필요할 것이라 생각 된다.

\section{Acknowledgements}

This work was supported by the National Research Foundation of Korea(NRF) grant funded by the Korean government(MEST) (No. 2011-0016753).

\section{References}

Bechtol, C., The use of a dynamometer with adjustable handle spacings, The Journal of Bone and Joint Surgery, 36, 820-832, 1954.

Eksioglu, M., Relative optimum grip span as a function of hand anthropometry, International Journal of Industrial Ergonomics, 34, 1-12, 2004.

Farris, B.A., Fernandez, J.E. and Agarwal, R.K., The effects of wrist posture on the force exerted by individual finger. In: B. Das and W. Karwowski eds. Proceedings of the advances in occupational ergonomics and safety II, 1-4 June 1997, Washington D.C.: IOS Press, 301-304, 1997.

Fransson, C. and Winkel, J., Hand strength: the influence of grip span and grip type, Ergonomics, 34, 881-892, 1991.

Greenberg, L. and Chaffin, D., Workers and their tools, Midland, MI: Pendell Publishin, 1977.

Hazelton, F.T., Gary L.S., Andrean E.F. and Ralph, I.S., The influence of the wrist position on the force produced by the finger flexors, Journal of Biomechanics, 8, 301-306, 1975.

Kim, D.M. and Kong, Y.K. , Development of the "Adjustable multi-finger force measurement (MFFM) systems" for research of handtool-related musculoskeletal disorders, Applied Human Factors and Ergonomics, 2nd International Conference, July 14-17, Las Vegas, USA, 2008.

Kong, Y.K., Kim, D.M., Lee, K.S. and Jung, M.C., Handle evaluation for young and old age groups by Multi-Finger Force Measurement (MFFM) system, 17th World Congress on Ergonomics, IEA (International Ergonomics Association), August 9-14, Beijing, CHINA, 2009.

Kong, Y.K., Kim, D.M., Lim, C.M. and Seo, M.T., Development of DFM (Double-Handle Force Measurement) System for Measuring Individual Finger Forces, Fall Conference and General Meeting of Ergonomics Society of Korea, October 21-22, Cheon-an, Korea, 2011.

Lee, S.J., Kong, Y.K., Lowe, B.D. and Song, S., Handle grip span for optimising finger-specific force capability as a function of hand size. Ergonomics, 52(5), 601-608, 2009.

Petersen, P., Petrich, M., Connor, H. and Conklin, D., Grip strength and hand dominance: challenging the $10 \%$ rule, The American Journal of Occupational Therapy, 43(7), 444-447, 1989.

Petrofsky, J., The effect of handgrip on isometric exercise performance. Ergonomics, 23, 1129-1135, 1980.

Talsania, J. and Kozin, S., Normal digital contribution to grip strength 
assessed by a computerized digital dynamometer. The Journal of Hand Surgery: Journal of the British Society for Surgery of the Hand, 23(2), 162-166, 1998.

\section{Author listings}

Yong-Ku Kong: ykong@skku.edu

Highest degree: $\mathrm{PhD}$, Industry Engineering, the Pennsylvania State University

Position title: Professor, Department of Industrial Engineering, Sungkyunkwan University

Areas of interest: Ergonomic Product Design, Hand Tool Design, Musculoskeletal Disorders

Jin Woo Jung: goleats@skku.edu

Highest degree: B.S., Department of Systems Management Engineering, Sungkyunkwan University

Position title: M.S. Candidate, Department of Industrial Engineering, Sungkyunkwan University

Areas of interest: Digital Virtual Manufacturing/PLM, Concurrent \& Collaborative Engineering, e-Manufacturing, Sustainable Manufacturing, Manufacturing System and Information

Sangmin Kim: kmjzx@skku.edu

Highest degree: B.S., Department of Systems Management Engineering, Sungkyunkwan University

Position title: M.S, Department of Industrial Engineering, the University of Sungkyunkwan

Areas of interest: Human-Computer Interaction, Accessibility, User Interface, User Experience, Universal Design
Heewoong Jung: Jeong8607@gmail.com

Highest degree: B.S., Department of Systems Management Engineering, Sungkyunkwan University

Position title: Student, Department of Industrial Engineering,

Sungkyunkwan University

Areas of interest: Human-Computer Interaction, User Interface, User Experience

Hakje Yoo: waihjei@hanmail.net

Highest degree: B.S., Department of Biomechatronics Engineering, Sungkyunkwan university

Position title: M.S. Candidate, Department of Biomedical Engineering, Sungkyunkwan university

Areas of interest: Motion Capture Analysis, Sports Biomechanics

Dae-Min Kim: kimdaemin@skku.edu

Highest degree: M.S., Department of Systems Management Engineering, Sungkyunkwan University

Position title: Ph.D. Candidate, Department of Industrial Engineering, Sungkyunkwan University

Areas of interest: Ergonomic Product Design, Musculoskeletal Disorders

Hyun-Sung Kang: kanghs328@gmail.com

Highest degree: B.S., Department of Systems Management Engineering, Sungkyunkwan University

Position title: M.S. Candidate, Department of Industrial Engineering, Sungkyunkwan University

Areas of interest: Musculoskeletal Simulation, Motion Capture Analysis, Bioelectric Signal Analysis, Ergonomic Product Design, Hand tools

Date Received : 2013-11-15

Date Revised : 2013-12-05

Date Accepted : 2013-12-10 